PROCEEDINGS OF THE

AMERICAN MATHEMATICAL SOCIETY

Volume 127, Number 11, Pages 3237-3242

S 0002-9939(99)04896-0

Article electronically published on April 28, 1999

\title{
NILPOTENT IDEALS IN A CLASS OF BANACH ALGEBRAS
}

\author{
YONG ZHANG
}

(Communicated by Theodore W. Gamelin)

\begin{abstract}
We introduce the concepts of approximately complemented subspaces of normed spaces and approximately biprojective algebras. We prove that any approximately biprojective Banach algebra with left and right approximate identities does not have a nontrivial nilpotent ideal whose closure is approximately complemented.
\end{abstract}

It is known that nonzero nilpotent ideals in amenable Banach algebras, if any, must be infinite dimensional [3], and there do exist amenable Banach algebras which contain nonzero nilpotent ideals (see [9] and [13]). These properties lead to the following natural question: Can a contractible Banach algebra contain a nonzero nilpotent ideal? Here a contractible Banach algebra is a biprojective Banach algebra with an identity, and a Banach algebra $\mathcal{A}$ is biprojective if and only if the continuous bimodule morphism $\pi: \mathcal{A} \hat{\otimes} \mathcal{A} \rightarrow \mathcal{A}$, specified by $\pi(a \otimes b)=a b$, has a continuous bimodule morphism right inverse $T: \mathcal{A} \rightarrow \mathcal{A} \hat{\otimes} \mathcal{A}$, i.e. $\pi \circ T=I_{\mathcal{A}}$, the identity operator on $\mathcal{A}$, where $\mathcal{A} \hat{\otimes} \mathcal{A}$ denotes the projective tensor product. One can see [12] for further properties of biprojective Banach algebras and [7], VII §1.4, for conditions equivalent to contractibility of Banach algebras.

In [9] Loy and Willis have proved that if a biprojective Banach algebra has a central approximate identity, then no nonzero nilpotent ideal can have the approximation property. In this paper we will improve this theorem by introducing the concepts of "approximately complemented subspaces" and "approximately biprojective algebras". We use some of the techniques in [9].

\section{Approximately COMPlemented Subspaces}

We first introduce the following:

Definition 1. Suppose that $E$ is a subspace of a normed space $X$. Then $E$ is approximately complemented in $X$ if for any compact subset $K$ of $E$ and any $\varepsilon>0$, there is a continuous operator $P: X \rightarrow E$ such that $\|x-P(x)\|<\varepsilon$, for all $x \in K$.

The following examples explain the use of the terminology "approximately complemented". First, if a subspace $E$ of $X$ is such that for any compact subset $K$ of $E$ there is a subspace $N$, complemented in $X$, such that $K \subset N \subset E$, then $E$

Received by the editors September 24, 1997 and, in revised form, January 23, 1998.

1991 Mathematics Subject Classification. Primary 46H10; Secondary 46H20, 46B25, 46A32.

Key words and phrases. Nilpotent ideals, approximately complemented, approximately biprojective, amenable Banach algebras, contractible Banach algebras.

(C)1999 American Mathematical Society 
is approximately complemented. In particular, any complemented subspace is approximately complemented. A subspace with the approximation property is also an approximately complemented subspace because any finite rank bounded operator on the subspace can be extended to the whole of the space. Another interesting example is that when $E$ is a right (left) ideal of a Banach algebra $\mathcal{A}$ and has a left (resp. right) bounded approximate identity, then $E$ is approximately complemented. In fact, suppose that $\left\{e_{\alpha}\right\}$ is a left bounded approximate identity of the right ideal $E$. Then for any compact $K \subset E$ and $\varepsilon>0$, there is an index $\alpha$ such that $\left\|x-e_{\alpha} x\right\|<\varepsilon$ for all $x \in K$. So $P: X \rightarrow E$ defined by $P(x)=e_{\alpha} x$ satisfies the requirement in Definition 1 . For $C^{*}$-algebras, it is well known that any closed left ideal has a bounded right approximate identity (see [8], Proposition 4.2.12). This shows that every closed left ideal of a $C^{*}$-algebra is approximately complemented. For any Banach space $X$, let $B(X)$ be the Banach algebra of all bounded operators on $X$ with the multiplication of the operator composition, and let $B_{K}(X)$ be the ideal of all compact operators on $X$. From [10], Theorem 5.1.12(d), $B_{K}(X)$ has a left bounded approximate identity if $X$ has the bounded compact approximation property. So $B_{K}(X)$ is approximately complemented in $B(X)$ if $X$ has this property. It is worth mentioning that, $B_{K}(X)$ is not complemented in $B(X)$ when $X$ is an infinite dimensional Hilbert space (see [2]).

Suppose that $X$ and $Y$ are normed spaces. Denote by $X \hat{\otimes} Y$ (resp. $X \check{\otimes} Y$ ) the projective (resp. injective) tensor product of $X$ and $Y$. We will use $\|\cdot\|_{X, Y}$ to denote the projective tensor norm in case any confusion may occur.

Suppose that $X_{1}, X_{2}, Y_{1}$ and $Y_{2}$ are normed spaces, and $T_{i}: X_{i} \rightarrow Y_{i}, i=1,2$, are bounded operators. We denote by $T_{1} \otimes T_{2}: X_{1} \hat{\otimes} X_{2} \rightarrow Y_{1} \hat{\otimes} Y_{2}$ the linear operator determined by

$$
\left(T_{1} \otimes T_{2}\right)\left(a_{1} \otimes a_{2}\right)=T_{1}\left(a_{1}\right) \otimes T_{2}\left(a_{2}\right), \quad\left(a_{1} \in X_{1}, a_{2} \in X_{2}\right) .
$$

Clearly, $T_{1} \otimes T_{2}$ is continuous and $\left\|T_{1} \otimes T_{2}\right\| \leq\left\|T_{1}\right\|\left\|T_{2}\right\|$. If

$$
A_{1} \stackrel{T_{1}}{\longrightarrow} B_{1} \stackrel{S_{1}}{\longrightarrow} C_{1} \quad \text { and } \quad A_{2} \stackrel{T_{2}}{\longrightarrow} B_{2} \stackrel{S_{2}}{\longrightarrow} C_{2}
$$

are bounded operator sequences of normed spaces, then we have

$$
\left(S_{1} \circ T_{1}\right) \otimes\left(S_{2} \circ T_{2}\right)=\left(S_{1} \otimes S_{2}\right) \circ\left(T_{1} \otimes T_{2}\right) .
$$

Lemma 1. Suppose that $X$ is a normed space and $E$ is an approximately complemented subspace of $X$. Let $\imath: E \rightarrow X$ be the inclusion mapping. Then $\imath \otimes I_{Y}: E \hat{\otimes} Y \rightarrow X \hat{\otimes} Y$ is injective for any normed space $Y$, where $I_{Y}$ is the identity mapping on $Y$.

Proof. Let $u \in E \hat{\otimes} Y$ satisfying $\imath \otimes I_{Y}(u)=0$ in $X \hat{\otimes} Y$. We prove $u=0$ in $E \hat{\otimes} Y$, or equivalently, $\|u\|_{E, Y}=0$. It is elementary that for every convergent series $\sum_{n=1}^{\infty} x_{n}$, $x_{n}>0$, there exists a sequence $\left(c_{n}\right)$ such that $c_{n} \rightarrow \infty$ and $\sum_{n=1}^{\infty} c_{n} x_{n}$ converges. Thus, without loss of generality, we can assume $u=\sum_{n=1}^{\infty} a_{n} \otimes b_{n}$ with $a_{n} \in E$ and $b_{n} \in Y, n=1,2, \ldots$, satisfying $\lim _{n \rightarrow \infty} a_{n}=0, \sum_{n=1}^{\infty}\left\|b_{n}\right\|<1$. Given $\varepsilon>0$, let $P: X \rightarrow E$ be a continuous operator satisfying $\left\|a_{n}-P\left(a_{n}\right)\right\|<\varepsilon$ for all $n$. Then $P \otimes I_{Y}: X \hat{\otimes} Y \rightarrow E \hat{\otimes} Y$ is a continuous operator, and hence

$$
\sum_{n=1}^{\infty} P\left(a_{n}\right) \otimes b_{n}=\left(P \otimes I_{Y}\right) \circ\left(\imath \otimes I_{Y}\right)(u)=0 .
$$


Therefore,

$$
\begin{aligned}
\|u\|_{E, Y} & =\left\|\sum_{n=1}^{\infty} a_{n} \otimes b_{n}-\sum_{n=1}^{\infty} P\left(a_{n}\right) \otimes b_{n}\right\|_{E, Y} \\
& \leq \sum_{n=1}^{\infty}\left\|a_{n}-P\left(a_{n}\right)\right\|\left\|b_{n}\right\|<\varepsilon
\end{aligned}
$$

Since $\varepsilon$ was arbitrary, we have $\|u\|_{E, Y}=0$, and hence $\imath \otimes I_{Y}$ is injective.

Remark. One cannot expect that $\imath \otimes I_{Y}$ will be injective for any closed subspace $E$ of $X$. In fact, if $X$ is a Banach space with the approximation property and $E$ is a closed subspace of $X$ without this property, then there is a Banach space $Y$ such that $\imath \otimes I_{Y}: E \hat{\otimes} Y \rightarrow X \hat{\otimes} Y$ has a nontrivial kernel. Because (see [6], p.156), if $E$ does not have the approximation property, then there is a Banach space $Y$ such that a nonzero element $u=\sum_{n=1}^{\infty} a_{n} \otimes b_{n} \in E \hat{\otimes} Y$ exists with $\sum_{n=1}^{\infty} a_{n} \otimes b_{n}=0$ in $E \check{\otimes} Y$. Since $E \check{\otimes} Y \subset X \check{\otimes} Y, \sum_{n=1}^{\infty} a_{n} \otimes b_{n}=0$ in $X \check{\otimes} Y$. This implies $\sum_{n=1}^{\infty} a_{n} \otimes b_{n}=0$ in $X \hat{\otimes} Y$, since $X$ has the approximation property. In other words, $\imath \otimes I_{Y}(u)=0$. The problem of under what condition $\imath \otimes I_{Y}$ is injective is still open.

Corollary. Suppose that $T_{i}: A_{i} \rightarrow B_{i}, i=1,2$, are topologically isomorphic embeddings (injective and homeomorphic) of Banach spaces. If $T_{i}\left(A_{i}\right)$ is approximately complemented in $B_{i}, i=1,2$, then the operator $T_{1} \otimes T_{2}$ is injective.

Proof. Let $\hat{T}_{i}: A_{i} \rightarrow T_{i}\left(A_{i}\right), i=1,2$, be the canonical isomorphisms induced from $T_{i}$, and $\imath_{i}: T_{i}\left(A_{i}\right) \rightarrow B_{i}, i=1,2$, be the inclusion mappings. Then

$$
T_{1} \otimes T_{2}=\left(I_{B_{1}} \otimes \imath_{2}\right) \circ\left(I_{B_{1}} \otimes \hat{T}_{2}\right) \circ\left(\imath_{1} \otimes I_{A_{2}}\right) \circ\left(\hat{T}_{1} \otimes I_{A_{2}}\right) .
$$

$I_{B_{1}} \otimes \hat{T}_{2}$ and $\hat{T}_{1} \otimes I_{A_{2}}$ are injective because they are invertible. From the condition and the previous lemma $I_{B_{1}} \otimes \imath_{2}$ and $\imath_{1} \otimes I_{A_{2}}$ are also injective. Subsequently, as a composition of injective operators $T_{1} \otimes T_{2}$ is injective.

\section{Approximately Biprojective Banach algebras}

In this section we introduce the following notion.

Definition 2. A Banach algebra $\mathcal{A}$ is pointwise approximately biprojective if for each $a \in \mathcal{A}$ there is a net $\left\{T_{\alpha} \mid \alpha \in \Lambda\right\}$ of continuous bimodule morphisms from $\mathcal{A}$ into $\mathcal{A} \hat{\otimes} \mathcal{A}$ such that $\pi \circ T_{\alpha}(a) \rightarrow a$ in norm. If in addition, the net $\left\{T_{\alpha} \mid \alpha \in \Lambda\right\}$ is independent of the element $a \in \mathcal{A}$, then $\mathcal{A}$ is approximately biprojective.

Of course any biprojective Banach algebra is approximately biprojective. Also, if $\mathcal{A}$ has a central approximate diagonal (i.e., a net $\left\{u_{\alpha} \mid \alpha \in \Lambda\right\} \subset \mathcal{A} \hat{\otimes} \mathcal{A}$ such that $a u_{\alpha}=u_{\alpha} a$ for all $a \in \mathcal{A}$ and $\alpha \in \Lambda$, and $\pi\left(a u_{\alpha}\right) \rightarrow a$ in norm), then $\mathcal{A}$ is approximately biprojective. An example of this kind of Banach algebra is as follows.

Example. Let $S$ be any nonempty set and consider $\ell^{2}(S)$ with the pointwise multiplication. Let $\Lambda$ be the collection of all finite subsets of $S$ ordered by inclusion. Then $\left\{u_{\alpha}=\sum_{i \in \alpha} e_{i} \otimes e_{i} \mid \alpha \in \Lambda\right\}$ forms a central approximate diagonal of $\ell^{2}(S)$, where $e_{i}$ is the element of $\ell^{2}(S)$ equal to 1 at $i$ and 0 elsewhere. We prove that $\ell^{2}(S)$ is not biprojective whenever $S$ is an infinite set. Suppose conversely that $T: \ell^{2}(S) \rightarrow \ell^{2}(S) \hat{\otimes} \ell^{2}(S)$ were a continuous bimodule morphism satisfying $\pi \circ T=I_{\ell^{2}(S)}$. Since $T\left(e_{i}\right)=e_{i} T\left(e_{i}\right) e_{i}$, one easily sees that $T\left(e_{i}\right)=e_{i} \otimes e_{i}$ for 
each $i \in S$. So if $x=\sum_{i \in S} \alpha_{i} e_{i} \in \ell^{2}(S)$, then $T(x)=\sum_{i \in S} \alpha_{i} e_{i} \otimes e_{i}$. Consider the identity operator $\imath \in B L\left(\ell^{2}(S), \ell^{2}(S)\right)$, which can be viewed as an element of $\left(\ell^{2}(S) \hat{\otimes} \ell^{2}(S)\right)^{*}$, when the latter is identified with $B L\left(\ell^{2}(S), \ell^{2}(S)\right)$ (cf. $\S 30$ of [1]). We have

$$
|\langle\imath, T(x)\rangle| \leq\|\imath\|\|T\|\|x\|=\|T\|\|x\| .
$$

But it is clear that $\left\langle\imath, e_{i} \otimes e_{i}\right\rangle=1$ for each $i \in S$, and so

$$
\langle\imath, T(x)\rangle=\sum_{i \in S} \alpha_{i}
$$

Together with the preceding inequality, one is led to a contradiction that $\sum_{i \in S} \alpha_{i}$ converges for each $x=\sum_{i \in S} \alpha_{i} e_{i} \in \ell^{2}(S)$. This example also shows that, for any commutative compact group $G, L^{2}(G)$ with convolution multiplication is approximately biprojective but is not biprojective unless $G$ is finite, because from Plancherel's Theorem ([11], Theorem 1.6.1) $L^{2}(G)$ is isometrically isomorphic to $\ell^{2}(\Gamma) ; \Gamma$ being the dual group of $G$ and $\ell^{2}(\Gamma)$ having pointwise multiplication. It is worth mentioning that, although $L^{2}(G)$ has a central approximate diagonal as shown here, it is not amenable unless $G$ is finite ([4], Proposition 1.10).

The terminology "approximately projective" was first used by Grønbæk for modules in [5], where that kind of modules were defined in terms of lifting of morphisms.

\section{MAIN RESUltS ON NILPOTENT IDEALS}

In the following for two subsets $M$ and $N$ of an algebra $\mathcal{A}, M N$ always denotes the set $\{m n \mid m \in M, n \in N\}$.

Lemma 2. Suppose that $\mathcal{A}$ is a pointwise approximately biprojective Banach algebra, $\mathcal{N}$ is an approximately complemented closed ideal of $\mathcal{A}$, and $E$ is a closed ideal of $\mathcal{A}$ satisfying $E \subseteq \mathcal{N}$ and $E \mathcal{N}=\{0\}(\mathcal{N} E=\{0\})$. Then for any subset $M$ of $\mathcal{A}$ satisfying $M E \subseteq \overline{E \mathcal{A}}$ (resp. $E M \subseteq \overline{\mathcal{A} E}$ ), it is true that $M E=\{0\}$ (resp. $E M=\{0\})$.

Proof. We prove the case $E \mathcal{N}=\{0\}$ and $M E \subseteq \overline{E \mathcal{A}}$. Here the idea is, in fact, from the proof of Proposition 1 of [9]. Let $\imath: \mathcal{N} \rightarrow \mathcal{A}$ be the inclusion operator, let $q: \mathcal{A} \rightarrow \mathcal{A} / E$ be the quotient operator, let $I_{\mathcal{A}}, I_{\mathcal{N}}$ and $I_{\mathcal{A} / E}$ be the identity operators on $\mathcal{A}, \mathcal{N}$ and $\mathcal{A} / E$ respectively, and let $p:(\mathcal{A} / E) \hat{\otimes} \mathcal{N} \rightarrow \mathcal{N}$ be the operator determined by $p((a+E) \otimes c)=a c$ for any $a+E \in \mathcal{A} / E$ and $c \in \mathcal{N}$. The operator $p$ is well defined because $E \mathcal{N}=\{0\}$. All the operators above are obviously continuous.

Suppose towards a contradiction that $M E \neq\{0\}$, and assume $m c \neq 0$ for some $m \in M, c \in E$. From the assumption $m c=\lim c_{n} a_{n}$, for some sequences $\left(c_{n}\right) \subset$ $E$ and $\left(a_{n}\right) \subset \mathcal{A}$. Since $\mathcal{A}$ is pointwise approximately biprojective, there is a continuous bimodule morphism $T: \mathcal{A} \rightarrow \mathcal{A} \hat{\otimes} \mathcal{A}$ such that $\pi \circ T(m) \cdot c \neq 0$. For $b \in \mathcal{N}$, let $R_{b}\left(L_{b}\right): \mathcal{A} \rightarrow \mathcal{N}$ be the map of right (resp. left) multiplication by $b$. We now consider the element $d \in(\mathcal{A} / E) \hat{\otimes} \mathcal{N}$ defined by

$$
d=\left(q \otimes R_{c}\right) \circ T(m) .
$$

Since

$$
p(d)=R_{c} \circ \pi \circ T(m)=\pi \circ T(m) \cdot c \neq 0,
$$


we have $d \neq 0$. Now consider its image under $I_{\mathcal{A} / E} \otimes \imath$.

$$
\begin{aligned}
\left(I_{\mathcal{A} / E} \otimes \imath\right)(d) & =\left(I_{\mathcal{A} / E} \otimes \imath\right) \circ\left(q \otimes I_{\mathcal{N}}\right) \circ\left(I_{\mathcal{A}} \otimes R_{c}\right) \circ T(m) \\
& =\left(q \otimes I_{\mathcal{A}}\right) \circ\left(I_{A} \otimes \imath\right) \circ\left(I_{\mathcal{A}} \otimes R_{c}\right) \circ T(m) \\
& =\left(q \otimes I_{\mathcal{A}}\right)(T(m) c) \\
& =\left(q \otimes I_{\mathcal{A}}\right) T(m c) \\
& =\lim \left(q \otimes I_{\mathcal{A}}\right) T\left(c_{n} a_{n}\right) \\
& =\lim \left(q \otimes I_{\mathcal{A}}\right) \circ\left(\imath \otimes I_{\mathcal{A}}\right) \circ\left(L_{c_{n}} \otimes I_{\mathcal{A}}\right) \circ T\left(a_{n}\right) \\
& =\lim \left(\left(q \circ \imath \circ L_{c_{n}}\right) \otimes I_{\mathcal{A}}\right) \circ T\left(a_{n}\right)=0,
\end{aligned}
$$

since $q \circ \imath \circ L_{c_{n}}=0$ as an operator from $\mathcal{A}$ to $\mathcal{A} / E$. But $I_{\mathcal{A} / E} \otimes \imath$ is injective due to Lemma 1 . Therefore the above equality implies that $d=0$, a contradiction which shows that $M E=\{0\}$.

Theorem. Suppose that $\mathcal{A}$ is a pointwise approximately biprojective Banach algebra. If $\mathcal{A}$ has both left and right approximate identities, then $\mathcal{A}$ possesses no nonzero nilpotent ideal whose closure is approximately complemented in $\mathcal{A}$.

Proof. If there were a nonzero nilpotent ideal $\mathcal{N}$ whose closure is approximately complemented, then there would be an integer $n \geq 1$ such that $\mathcal{N}^{n} \neq\{0\}$ and $\mathcal{N}^{n+1}=\{0\}$. Without loss of generality, assume that $\mathcal{N}$ is closed. Let $E$ be the closure of the linear span of $\mathcal{N}^{n}$. Then $E$ would be a nonzero closed ideal of $\mathcal{A}$ satisfying $E \subset \mathcal{N}$ and $E \mathcal{N}=\{0\}$. Since $\mathcal{A}$ has a left approximate identity, $E=\overline{\mathcal{A} E}$. Similarly $E=\overline{E \mathcal{A}}$. So $\mathcal{A} E \subseteq \overline{E \mathcal{A}}$. Then $\mathcal{A} E=0$, from Lemma 2, which leads to a contradiction that $E=\overline{\mathcal{A E}}=\{0\}$.

Remark. The requirement of the existence of both left and right approximate identities in the above theorem cannot be reduced to the existence of just one one-sided approximate identity, as seen from the following example of Helemskii (see [9]). The Banach algebra of $2 \times 2$ matrices with zero first column is biprojective. It has a right identy $\left(\begin{array}{ll}0 & 0 \\ 0 & 1\end{array}\right)$ but no left (approximate) identity, and it does have a nonzero nilpotent ideal (which must be complemented since the algebra is finite dimensional) containing all the matrices of the form $\left(\begin{array}{ll}0 & a \\ 0 & 0\end{array}\right)$.

Corollary 1. A pointwise approximately biprojective Banach algebra with both left and right approximate identities has no nonzero finite dimensional nilpotent ideals.

Proof. Any finite dimensional subspace is complemented.

Corollary 2. Suppose that $\mathcal{A}$ is a pointwise approximately biprojective Banach algebra whose underlying space is a Hilbert space. If $\mathcal{A}$ has both left and right approximate identities, then $\mathcal{A}$ possesses no nonzero nilpotent ideals. If in addition, $\mathcal{A}$ is commutative, then it has no nontrivial nilpotent elements.

Proof. Every closed subspace of a Hilbert space is complemented. If in addition, $\mathcal{A}$ is commutative, then the ideal generated by a nilpotent element is nilpotent.

Since contractible Banach algebras are biprojective and have identities ([7], VII.1.72), we can immediately deduce the following results. 
Corollary 3. Suppose that $\mathcal{A}$ is a contractible Banach algebra. Then $\mathcal{A}$ possesses no nonzero nilpotent ideals which are approximately complemented.

Remark. Corollary 2 is not new for the case that $\mathcal{A}$ is contractible. In fact, a contractible Banach algebra whose underlying space is a Hilbert space is finite dimensional (see [4], Proposition 1.10) and hence is isomorphic to a Cartesian product of a finite number of complete matrix algebras. Since each complete matrix algebra (which is always simple) is not nilpotent, any nonzero ideal of $\mathcal{A}$ (which must also be isomorphic to a Cartesian product of a finite number of complete matrix algebras) is not nilpotent.

Similar results can be obtained for Banach algebras with central approximate diagonals. For example we have:

Corollary 4. Suppose that $\mathcal{A}$ is a Banach algebra with a central approximate diagonal. Then $\mathcal{A}$ possesses no nonzero nilpotent ideal which is approximately complemented.

\section{ACKNOWLEDGEMENT}

The author would like to thank Professor F. Ghahramani who introduced this topic to him and, during the preparation of this paper, gave him many valuable suggestions. He also thanks Professor J. P. McClure for bringing the monograph [6] to his attention.

\section{REFERENCES}

[1] F. F. Bonsall and J. Duncan, Complete Normed Algebras, Springer-Verlag, Berlin, Heidelberg, New York, 1973. MR 54:11013

[2] J. B. Conway, The compact operators are not complemented in $\mathcal{B}(\mathcal{H})$, Proc. Amer. Math. Soc. 32 (1972), 549-550. MR 44:5156

[3] P. C. Curtis, Jr., and R. J. Loy, The structure of amenable Banach algebras, J. London Math. Soc. 40 (1989), 89-104. MR 90k:46114

[4] F. Ghahramani, R. J. Loy, and G. A. Willis, Amenability and weak amenability of second conjugate Banach algebras, Proc. Amer. Math. Soc. 124 (1996), 1489-1497. MR 96g:46036

[5] N. Grønbæk, Morita equivalence for Banach algebras, J. Pure and Applied Algebra 99 (1995), 183-219. MR 96e:46099

[6] A. Grothendieck, Produits Tensoriels Topologiques et Espaces Nucléaires, Mem. Amer. Math. Soc., No.16, 1955. MR 76:763c

[7] A. Ya. Helemskii, Banach and Locally Convex Algebras, Oxford University Press, Oxford, New York, Toronto, 1993. MR 94f:46001

[8] R. V. Kadison and J. R. Ringrose, Fundamentals of the Theory of Operator Algebras, Vol. I, Academic Press, Orlando, Florida, 1986. MR 88d:46106

[9] R. J. Loy and G. A. Willis, The approximation property and nilpotent ideals in amenable Banach algebras, Bull. Austral. Math. Soc. 49 (1994), 341-346. MR 94m:46083

[10] T. W. Palmer, Banach Algebra and the General Theory of *algebras, Vol. I, Cambridge University Press, 1994. MR 95c:46002

[11] W. Rudin, Fourier Analysis on Groups, Interscience Publishers, New York - London, 1962. MR 27:2808

[12] Yu. V. Selivanov, Biprojective Banach algebras, Math USSR Izvestija 15 (1980), 387-399.

[13] N. Th. Varopoulos, Spectral synthesis on spheres, Proc. Cambridge Philos. Soc. 62 (1966), 379-387. MR 34:1786

Department of Mathematics and Astronomy, University of Manitoba, Winnipeg, ManITOBA, CANADA R3T 2N2

E-mail address: umzhan20@cc.umanitoba.ca 